Voix et Images

voixetimages

\title{
Fils déchus et autres matières
}

\section{André Brochu}

Volume 31, numéro 2 (92), hiver 2006

Gilles Archambault

URI : https://id.erudit.org/iderudit/012884ar

DOI : https://doi.org/10.7202/012884ar

Aller au sommaire du numéro

Éditeur(s)

Université du Québec à Montréal

ISSN

0318-9201 (imprimé)

1705-933X (numérique)

Découvrir la revue

Citer cet article

Brochu, A. (2006). Fils déchus et autres matières. Voix et Images, 31(2), 159-164.

https://doi.org/10.7202/012884ar

Ce document est protégé par la loi sur le droit d'auteur. L'utilisation des services d'Érudit (y compris la reproduction) est assujettie à sa politique d'utilisation que vous pouvez consulter en ligne.

https://apropos.erudit.org/fr/usagers/politique-dutilisation/ 


\section{P O É S I E}

Fils déchus et autres matières
$++_{+}$

ANDRÉ BROCHU

Université de Montréal

Jamais correspondance n'aura mieux porté son nom. La Conversation poétique entre Harry Bernard et Alfred DesRochers ${ }^{1}$ est affaire de parole vive, d'échange quasi verbal entre deux accros de la poésie qui non seulement confrontent leurs idées sur le sujet, mais s'envoient des poèmes par la tête, se louangent, se critiquent et bâtissent là-dessus une complicité admirable.

\section{UNE AMITIÉ SANS FAILLE}

On assiste à l'éclosion de l'amitié entre deux écrivains qui, à la fin des années 1920 et au début de la décennie qui suit, sont largement reconnus comme le meilleur romancier et le meilleur poète de leur époque. Et, curieusement, leurs échanges épistolaires correspondent exactement à la période où ils se révèlent et donnent le meilleur d'eux-mêmes, Bernard avec ses romans (en particulier les deux derniers, Juana mon aimée et Dolorès) et un recueil de nouvelles; DesRochers avec son seul grand livre, À l'ombre de l'Orford.

Les romans de Bernard seront commentés par DesRochers, même sur manuscrit. Seront aussi passées au crible les critiques, souvent acerbes, qui accueillent leur parution. Elles sont l'œuvre de blancs-becs comme Lucien Parizeau, mais aussi de pontes tel $\mathrm{M}^{\mathrm{gr}}$ Camille Roy, très à cheval sur des détails de formulation, ou de JeanCharles Harvey, d'Albert Pelletier, de Valdombre (Claude-Henri Grignon) et de quelques autres. En privé, DesRochers prend résolument le parti de Bernard, même s'il est peu porté vers la forme romanesque. Bernard reçoit des prix, mais peu de fleurs. On lui fait une réputation d'auteur banal et qui maîtrise mal sa langue. À partir de Juana mon aimée, les compliments prendront le dessus. Quant à DesRochers, il atteint la célébrité d'un coup, à trente ans, et s'y maintient, même en ne publiant plus. Bernard fait un peu figure d'obscur tâcheron à côté de lui, le favorisé de la gloire.

1 Harry Bernard et Alfred DesRochers, Conversation poétique, édition préparée par Micheline Tremblay et Guy Gaudreau, Ottawa, Les Éditions David, 2005, 384 p. 
Une fois posés les protagonistes, voyons ce qui se joue.

Bernard est romancier, certes, mais il écrit aussi des vers. Il en envoie à DesRochers, qui se montre enthousiaste. DesRochers est-il sincère? Il confie à son correspondant qui s'étonne de son admiration pour son amie Jovette-Alice Bernier:

Vous me dites ne pas comprendre mon engouement pour les vers de Mlle Bernier. Autrefois, j'avais votre intransigeance; mais aujourd'hui je ne veux avoir de sévérité que pour moi-même. À quoi ça sert d'être «honnête» en littérature? On est pris pour un lecteur d'anthologie. J'ai pour critère, à présent, de n'apprécier les vers qu'à travers mes amitiés. (86-87)

On peut en déduire que la même indulgence s'appliquera aux vers de Bernard et, pourquoi pas, à ses romans.

Pourtant, les accents de la sincérité imprègnent les éloges et, du reste, les remarques défavorables sur les écrits de son ami ne manquent pas. Alfred DesRochers se fait fort de pouvoir admirer une esthétique littéraire à l'opposé de la sienne, mais il ne se prive pas de souligner les faiblesses ou les défauts d'un texte. Harry Bernard adoptera la même attitude à l'égard de son correspondant.

Homme très civilisé, peu enclin à la polémique, travailleur acharné, Bernard est aux antipodes du bouillant DesRochers. Ce dernier clame très haut les vertus de la langue française et met à profit ses ressources dans son œuvre littéraire, mais dans sa correspondance il adopte une prose expressive et pas plus soignée qu'il ne faut. En poésie, DesRochers se pose comme un lyrique, non pas à la manière déliquescente de Charles Baudelaire (comme le lui reproche Bernard au début de leur relation), mais plutôt à la façon énergique et libre d'un Pierre Ronsard, qu'il vénère. À l'occasion, il se réclamera du romantisme, contrairement à Bernard qui répugne aux effusions rhétoriques.

Sur la base de cette opposition des «tempéraments» aussi bien que des poétiques, les deux hommes se réclament, momentanément du moins, d'une même doctrine littéraire, le régionalisme (ou le terroirisme). Or, le régionalisme, qui inspire les premiers romans de Bernard, servira plutôt mal celui-ci; alors que, après des débuts en poésie que certains ont pu qualifier de baudelairiens (c'est le cas, je l'ai dit, de Bernard), DesRochers transforme sa manière du tout au tout en écrivant ses sonnets d'À l'ombre de l'Orford. Loin de l'exotisme universaliste, DesRochers s'impose grâce à des représentations très réalistes de la campagne "canadienne». Avoir appliqué à ces sujets une forme rigoureuse, qu'on peut qualifier de post-parnassienne (sinon de symboliste, ce qui nous éloignerait de la rigueur), voilà le grand coup réussi par DesRochers, et jamais plus il ne retrouvera, dans son œuvre, un semblable alliage de magie réaliste et de vérité incantatoire. Le régionalisme, comme il l'a vécu, ne pouvait être que l'état de grâce très provisoire d'un "fils déchu de race surhumaine ${ }^{2}$ ", pour un instant converti à l'évangile des champs.

2 Alfred DesRochers, Euvres poétiques, t. I, édition établie par Romain Légaré, Montréal, Fides, coll. «Nénuphar», 1977, p. 77. 
Pour Bernard qui s'applique dans ses premiers romans à peindre le petit monde de la campagne, le terroirisme, qui ne va pas sans un vertueux dogmatisme, constitue une limite évidente à son inspiration. C'est son dépassement vers une littérature plus urbaine et psychologique, proche des exotistes, qui lui fera connaître un certain succès et qui le haussera au-dessus de lui-même. C'est dire que, pour les deux amis, le poète et le romancier, tout se passe à l'inverse de ce qu'on attendrait. DesRochers triomphe dans et par le régionalisme, qui lui est au départ étranger, et Bernard trouve le salut dans la récusation du terroir originaire. Et les deux, une fois parvenus au sommet de leur art, cessent d'écrire, comme si la perte de contact avec leur inspiration initiale leur était fatale. Ils ont donné le meilleur d'eux-mêmes dans ce qui leur était contraire, et ils ne peuvent plus renouer le fil de leur démarche.

La correspondance, soigneusement présentée, annotée et éditée, fait suivre fréquemment les lettres de poèmes parfois considérables, soumis à l'appréciation du destinataire. On est frappé par l'abondante inspiration de Bernard, qui affiche une grande modestie, conteste les éloges de DesRochers et résiste à l'injonction de réunir ses vers en recueil. Pourtant, ses vers ont souvent de l'originalité et de la force, dans le registre élégiaque et fin qu'un Albert Lozeau a pratiqué avant lui. Sans doute, on relève peu de continuité thématique d'un poème à l'autre, comme si l'auteur changeait chaque fois ses perspectives en quête d'un filon sûr. Le recueil que Bernard n'a jamais publié aurait eu des allures d'anthologie. Mais un métier attestant une solide formation littéraire donne du prix à ces essais, et DesRochers n'a certainement pas tort de clamer haut et fort leurs mérites. Pour lui, Bernard est l'un des rares écrivains du cru à savoir «faire le vers", fabriquer un alexandrin consistant.

Du côté de DesRochers, on est frappé par l'absence totale de la veine bucolique. Veaux, vaches, cochons, couvée sont, pour ainsi dire, hors champ... Des poèmes amoureux, peut-être autorisés par le grand exemple de Ronsard (que la religion n'étouffait pas), occupent beaucoup de place, et l'inspiration contamine un tant soit peu Bernard, d'emblée tout aussi catholique. Bref, on est aux antipodes de l'image qu'on se fait volontiers de deux écrivains régionalistes, qui ont pour credo: « Notre littérature doit être avant tout canadienne et catholique» (68), et s'il est vrai que DesRochers triomphe - brièvement - dans le sonnet rustique ou dans l'évocation nostalgique des surhommes d'autrefois, "[r]ace de violents, de forts, de hasardeux ${ }^{3}$ ", il n'en séjourne pas moins dans une sorte de poésie confidentielle qui offre peu de chance à l'expression de son tempérament épique. Elle annonce toutefois la tendresse sans façon du recueil de la vieillesse, Élégies pour l'épouse en-allée (1967).

La Conversation poétique, tant par sa prose que par ses vers, ouvre une fenêtre précieuse sur un moment de l'histoire du poème au Québec, moment où les contradictions sont sur le point de faire éclater une tradition encore vigoureuse, mais incapable d'harnacher une réalité qui, désormais et pour toujours, se fabrique en ville.

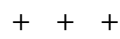


Mentionnons pour finir que, adeptes de la prosodie traditionnelle, DesRochers et Bernard sont passablement informés des nouvelles avenues de la littérature en France et dans le monde, et c'est en toute connaissance de cause qu'ils s'en tiennent au vers régulier. On peut voir là une raison de leur silence poétique (la remarque vaut surtout pour DesRochers), après une période d'intense création. Ils auront fait donner à la versification traditionnelle ce qu'elle pouvait donner, juste avant qu'une autre prosodie, mieux accordée au siècle des grands chambardements, prenne d'un coup toute la place.

\title{
DU MOI SENSIBLE AU MOI RESPONSABLE
}

Il n'y a pas de plus grande consécration, pour un poète québécois, que la publication de son œuvre dans la collection «Rétrospectives» de l'Hexagone. Seuls les meilleurs auteurs y ont droit. Pierre Nepveu a obtenu deux fois le Prix du Gouverneur général pour ses plus récents recueils de poésie, mais le couronnement véritable, c'est la parution du Sens du soleil ${ }^{4}$, qui réunit ses six recueils depuis Voies rapides (1971) jusqu'au célèbre Lignes aériennes (2002).

Grand intellectuel, essayiste remarquable qui a su situer la réalité québécoise dans celle de l'Amérique sans biaiser ni l'une ni l'autre, romancier fin et attaché à la substance des êtres, Pierre Nepveu est d'abord poète, et c'est par là qu'il commence, au début des années 1970, à jalonner sa trajectoire d'écrivain.

Les premiers recueils sont très riches de matière - pour employer un mot que Nepveu affectionne et qui se retrouve dans le beau titre du quatrième recueil, Mahler et autres matières. Ils expriment justement une sensibilité très grande et tout imprégnée de substances, de sensation, du pâteux quotidien, qu'il s'agisse d'ambiance urbaine ou de nature enveloppante, par exemple de la forêt. Cette matérialité a d'ailleurs quelque chose d'obsédant, souvent d'accablant. On dirait que Nepveu ne réussit son enracinement dans l'existence qu'au prix d'un exil qui le place à distance de la pensée ou des idées, et d'un éloignement du bonheur. Les sujets qu'il aborde sont à première vue banals, concernent la vie immédiate, comme le montre ce poème intitulē «Rentrée»:

\author{
on glisse sur de longues voies \\ plongeant leurs lames lumineuses \\ dans le nœud obscur où se terre la ville \\ fines traînées de feu s'enfoncent dans la nuit \\ et la nuit avale de tous ses boulevards inassouvis (30)
}

Il est rare qu'un poème élise comme objet d'une évocation la simple rentrée en ville, la nuit, en auto... Michel Beaulieu et Pierre Nepveu ont élargi la représentation poétique à ce genre de thèmes, ce qui supposait un recours au moi individuel dans

$$
++
$$

4 Pierre Nepveu, Le sens du soleil, Montréal, l’Hexagone, coll. «Rétrospectives», 2005, 468 p. 
sa vérité la plus nue. "Couleur chair», tel sera d'ailleurs le titre du troisième recueil de Nepveu ${ }^{5}$, et il faut y lire un parti pris de l'intimité matérielle en accord avec les spécifications les plus infimes de l'instant présent.

La rentrée en ville, donc; et c'est déjà le même climat, plein de bolides, d'avions, de lumières et de vitesse, de nuit ogresse aussi avec ses boulevards avides et son cœur noir, qu'on retrouvera dans Lignes aériennes. La modernité, haletante et sauvage. À ceci près que la tragédie de Mirabel, plus précisément de l'expropriation des terres sur laquelle le gouvernement canadien a construit l'aéroport, violant l'espace paysan au nom d'une rentabilité irréaliste, subordonne l'évocation de la modernité à celle de la blessure incicatrisable faite à l'humain. Le moi sensible du poète retraite alors devant le sens des responsabilités humaines, qui s'exprime à propos d'un gâchis énorme tout pénétré de la matérialité quotidienne. La vision carnavalesque du monde qu'on trouve dans Voies rapides et Épisodes, vision plus malaisée que vraiment désespérée, s'enrichit de raisons, de preuves, prend place dans un cadre réel, non pas générique comme la ville avec ses édifices et ses boulevards, mais précis, nommé: Mirabel. Un lieu devient le "Sphinx» (c'est le titre du poème), hanté qu'il est de tout un surnaturel souterrain, de la fatalité intemporelle:

\author{
Nous cherchons les traces \\ de ce qui est sans mémoire et sans voix \\ nous entrons aphasiques dans la cafétéria \\ où l'on ne sert plus de repas chauds, \\ absorbés par l'énigme de cet édifice aérien \\ ses immenses verrières sur ces pistes vides (384)
}

Admirons la sublimation du lieu le plus prosaïque qui soit, la cafétéria à moitié désaffectée, par l'évocation de l' «édifice aérien» auquel elle appartient, comme si l'aérogare était soudain un avion monumental et tout de verre, sur le point de décoller. Le mythe à la fois fascinant et menaçant (Sphinx) investit le présent, lieu de privation : l'on est privé de parole («aphasiques»), la fonction de restauration est supprimée ( «l'on ne sert plus de repas chauds»), on est mangé plutôt ( «absorbés par l'énigme»), un passé inquiétant, terrible, s'impose aux visiteurs.

D'un bout à l'autre de l'œuvre poétique de Nepveu, on est confronté à l'expression d'un même malaise devant la vie ou devant l'amour, d'une pente mélancolique très forte, d'un étonnement face à une quotidienneté chaotique, triviale, parfois obscène, mais aussi de courageux sursauts et de rappels des bonheurs simples, des raisons d'espérer. Dans les premiers recueils, cette expression est compulsive et pas très limpide, la syntaxe se modelant sur le chaos représenté; mais peu à peu, dans les suivants, elle s'harmonise avec une expérience plus poussée du

5 On trouvera des comptes rendus de Couleur chair et de Mahler et autres matières dans mon Tableau du poème. La poésie québécoise des années quatre-vingt, Montréal, XYZ éditeur, 1994, p. 77-78, et de Romans-fleuves dans mon article intitulé "Pierre Nepveu l'existeur», Les Écrits, n 93, 1998, p. 41-54. 
monde, laquelle ne perd rien en tristesse ou en gravité, mais est éclairée par la sagesse et la lucidité. Le paquet des impressions cède la place à une raison qui clarifie le langage, rend le poème plus accessible et le charge de dimensions de pensée, de connaissance mieux détaillées. Le poète responsable, même devant un gâchis comme Mirabel, ne se contente pas de blâmer, il cherche à comprendre tous les aspects d'une réalité sociale, humaine, naturelle, technique où se reflète la complexité du monde et de nos destins.

La poésie peut être aussi cela, quand un grand élan de générosité et de compassion contribue à son embrasement. 